\title{
Comportamiento a fatiga de mezclas asfálticas colombianas con adición de pavimento reciclado al $100 \%$
}

\section{Fatigue behavior of asphalt mixtures colombian with addition recy- cled pavement asphalt to $100 \%$}

Neidy Betssey Patiño Boyacá

Ingeniera civil, auxiliar de investigación del Grupo Geotecnia de la Universidad Militar Nueva Granada, Bogotá, Colombia.

Contacto: U1100865@unimilitar.edu.co

\section{Oscar JaVier Reyes-Ortiz}

Ingeniero civil, magíster en Ingeniería Civil, doctor en Ingeniería, profesor titular de la Universidad Militar Nueva Granada, Bogotá, Colombia.

Contacto: oscar.reyes@unimilitar.edu.co

\section{Javier Fernando Camacho-Tauta}

Ingeniero civil, magíster en Ingeniería Civil, doctor en Ingeniería, profesor asociado de la Universidad Militar Nueva Granada, Bogotá, Colombia.

Contacto: javier.camacho@unimilitar.edu.co

Fecha de recepción: 29 de agosto de 2013

Clasificación del artículo: investigación

Fecha de aceptación: 2 de mayo de 2014

Financiamiento: Vicerrectoría de Investigaciones de la Universidad Militar Nueva Granada

DOI: http://dx.doi.org/10.14483/udistrital.jour.tecnura.2015.1.a05

Palabras clave: asfalto, granulometría, ley de fatiga, pavimento asfalto reciclado (RAP), rigidez.

Keywords: fatigue law, gradation asphalt, recycled asphalt pavement (RAP) and stiffness.

\section{RESUMEN}

Esta investigación se desarrolló con el objetivo de establecer el comportamiento a fatiga de mezclas asfálticas con granulometrías MD20 del Instituto de Desarrollo Urbano (IDU) y MDC 2 del Instituto Nacional de Vías (Invias), con adición de granular reciclado de pavimento flexible (RAP) con asfaltos de penetración 60/70 y 80/100. La primera etapa consistió en caracterizar el asfalto y el RAP además de calcular el porcentaje óptimo de asfalto para adicionar a las mezclas con RAP $100 \%$. A continuación, se fabricaron probetas trapezoidales con las diferentes granulometrías y tipos de asfalto, para finalmente ser ensayadas a fatiga a diferentes deformaciones. Entre los resultados encontrados se determinó que las mezclas analizadas tienen una alta rigidez, aunque esta varía según el asfalto utilizado, y que la mezcla con 
mejor vida a fatiga respecto a las estudiadas es la MDC-2 con asfalto 80/100.

\section{ABSTRACT}

This research was conducted in order to establish the fatigue behavior of asphalt mixtures with gradations MD20 Instituto de Desarrollo Urbano (IDU) and MDC 2 of The Instituto Nacional de Vías (INVIAS), with addition of recycled granular flexible pavement (RAP) with asphalt of

\section{INTRODUCCIÓN}

Los pavimentos deteriorados se pueden recuperan mediante el proceso de fresado, y el material obtenido se puede usar para fabricar nuevas mezclas asfálticas, siendo esta una técnica económica y medioambiental muy rentable. Este procedimiento aprovecha el material desechado no renovable y genera la utilización de árido virgen en menor porcentaje (Marantzidis y Giraldo, 2011). En países europeos el uso de pavimentos asfálticos reciclados (RAP) es una práctica común. Según estudios de la EAPA, cada año se reutilizan y reciclan alrededor de 50 millones de toneladas (EAPA, 2008). Asimismo, en Estados Unidos consideran el reciclado de pavimentos en caliente como una práctica habitual, que presenta resultados satisfactorios cuando se ejerce un adecuado control en el proceso de fabricación (Nguyen, 2013; Abakovic et ál., 2010).

A pesar de que en varios países se ha implementado el uso de RAP, esto no se realiza en altos porcentajes, debido a su calidad y la influencia en la seguridad vial. En Estados Unidos, la National Asphalt Pavement Association (NAPA) afirma que las mezclas que contienen altos porcentajes de RAP (30-40\%) se pueden producir, aunque la restricción para su fabricación radica en que penetration $60 / 70$ and $80 / 100$. The first step was to characterize the asphalt and RAP as well as calculate the optimum percentage of asphalt to be added to $100 \%$ RAP mixtures. Then samples were fabricated with different trapezoidal gradations and asphalt, to be tested finally fatigue at different deformations. Among the results found, it was determined that the tested mixtures have a high stiffness; although, it varies depending on the asphalt mix used and that the best fatigue life with respect to those studied is the MDC-2 with bitumen 80/100.

*

la rigidez aumenta a mayor contenido de RAP (Newcomb, Brown y Epps, 2007). Otros investigadores encontraron que el $40 \%$ de RAP, es el contenido máximo permitido para resistir deformaciones permanentes y agrietamientos por fatiga (Chen, Chu y Lin, 2007).

El diseño de mezclas que contienen RAP pretende igualar la calidad de las formuladas con materiales procedentes de cantera; sin embargo, algunos estudios realizados en pavimentos con RAP muestran que el daño más frecuente es la fatiga (Reyes, Camacho y Vargas, 2012). Por esta razón, investigadores de la Universidad de Tennessee encontraron que el aumento de la fragilidad y la disminución del índice de dureza por la incorporación de RAP compromete la vida a fatiga de mezclas en caliente, ya que la energía requerida para fracturar estas mezclas disminuye a medida que aumenta el porcentaje de RAP (Shu, Huang y Vukusavljevic, 2008). Además, la Universidad Tecnológica de Hassan señala que la relación entre la resistencia a la fatiga y la rigidez depende del espesor de capa; por tanto, para las capas delgadas de pavimento, la resistencia a la fatiga disminuye cuando aumenta la rigidez (Hassan, 2009). Asimismo, el comportamiento a fatiga parece mejorar con el aumento en la proporción de rejuvenecedores, lo que per- 
mite aumentar el contenido de RAP sin cambiar el comportamiento de las mezclas, dando ciclos más largos de vida a fatiga y reduciendo la temperatura necesaria para tener una adecuada trabajabilidad (Widyatmoko, 2008; Silva, 2012; Tabaković, Gibney, Cilchrist y McNally, 2006; Colbert, y Zhanping, 2012).

Dado que el contenido de RAP en la fabricación de nuevas mezclas asfálticas es una de las principales variables en la resistencia a fatiga de la mezcla, investigadores de la Universidad Politécnica de Cataluña han realizado diferentes estudios experimentales para caracterizar el comportamiento mecánico de las mezclas asfálticas que contienen altos contenidos de RAP, y han encontrado que es posible utilizar hasta $60 \%$ de pavimento reciclado en fabricación de mezclas, sin modificar significativamente las leyes de fatiga con respecto a mezclas patrón (Miro, Valdés, Martínez, Segura y Rodríguez, 2011; Valdés, Pérez, Miro, Martínez y Botella, 2011). Asimismo, investigadores de la Universidad de La Coruña constatan que las mezclas asfálticas en caliente tipo gruesas, con 50\% de áridos reciclados, tienen una resistencia a la fatiga similar a las fabricadas con $100 \%$ de áridos de cantera (Pérez, Galelgo, Toledano, Taibo y Garrido, 2007).

Con base en investigaciones desarrolladas en el mundo, se planteó esta investigación, cuyo objetivo principal fue determinar el comportamiento a fatiga de mezclas asfálticas con curvas granulometrías MD-20 del Instituto de Desarrollo Urbano (IDU) (IDU, 2011) y MDC-2 del Instituto Nacional de Vías (Invias) (Invias, 2007), con adición de RAP al $100 \%$ y así plantear la posibilidad de usar RAP en altos porcentajes en Colombia, con el propósito de ayudar a un desarrollo sostenible, ya que esta práctica ha sido poco o nada empleada en el país.

\section{METODOLOGÍA Y MATERIALES}

La investigación se desarrolló siguiendo la metodología que se presenta en la figura 1, iniciando con la caracterización del RAP y de los asfaltos de penetración 60/70 y 80/100 utilizados en el estudio. Acto seguido, se determinó por centrifugación el contenido de asfalto del pavimento reciclado. Posteriormente, se obtuvo el valor óptimo de asfalto para adicionar a la mezcla con RAP $100 \%$, con base en los mejores valores de flujo, estabilidad y densidad de las probetas con granulometrías MD20 del IDU y MDC-2 del Invias en su franja media (ver figura 2). A continuación se fabricaron los especímenes y se ejecutaron los ensayos de fatiga con muestras trapezoidales, a diferentes niveles de deformación. Finalmente, se obtuvieron las leyes de fatiga y se establecieron conclusiones y recomendaciones del uso de RAP al $100 \%$ en las mezclas asfálticas del estudio.

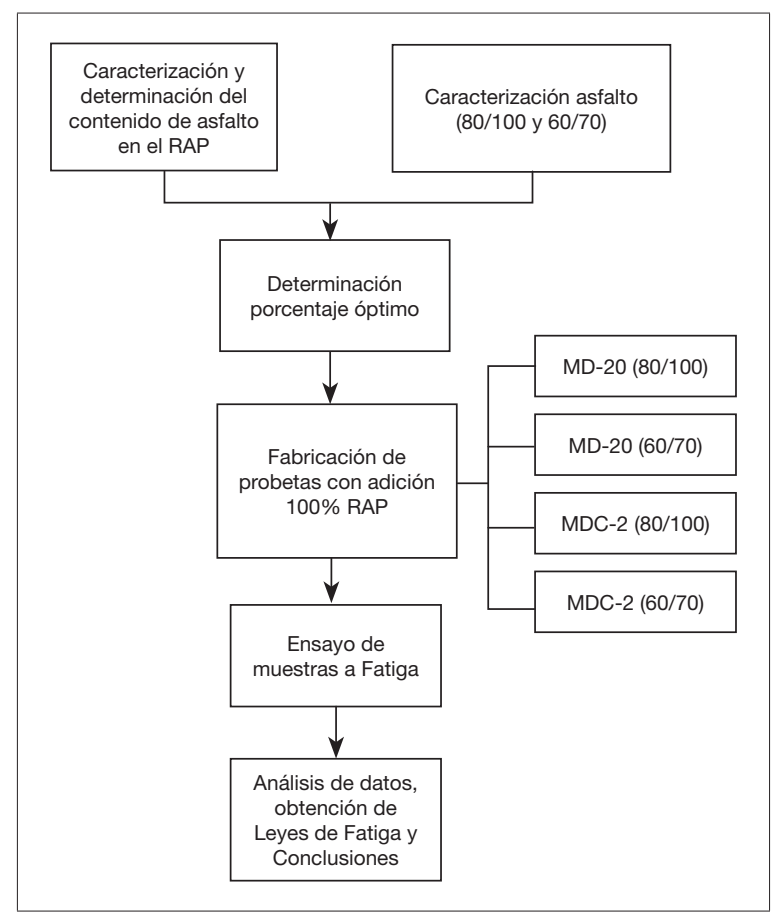

Figura 1. Diagrama de la metodología de la investigación

Fuente: elaboración propia. 


\section{Características del asfalto}

Las características de los asfaltos empleados en la investigación se relacionan en la tabla 1 .

\section{Contenido de asfalto de pavimentos asfálticos reciclados}

El contenido de asfalto que presentaba el pavimento reciclado se determinó mediante centrifugación, según la norma ASTM D-2172 (ASTM, 2013). Los resultados aparecen en la tabla 2. Se estableció que en promedio contiene $6,18 \%$.

\section{Diseño de mezcla con pavimentos asfálticos reciclados}

Para determinar el porcentaje que era preciso adicionar de asfalto para las mezclas fabricadas con RAP al $100 \%$, se fabricaron muestras con adición de 2, 3, 4 y 5\% de asfalto. En las figuras 3 y 4 se observan los resultados de estabilidad, flujo y densidad, parámetros estos que sirvieron para establecer el 3\% como óptimo.

Tabla 1. Ensayos de caracterización de los asfaltos

\begin{tabular}{|l|l|l|l|}
\hline \multicolumn{1}{|c|}{ Ensayo } & \multicolumn{1}{|c|}{ Norma } & \multicolumn{1}{c|}{ Asfalto 1 (60/70) } & \multicolumn{1}{c|}{ Asfalto 2 (80/100) } \\
\hline Penetración (1/10 mm) & ASTM D 5-97 & 63 & 89 \\
\hline Ductilidad (cm) & ASTM D 113-99 & 120 & 133 \\
\hline Viscosidad (poises) & ASTM D 2170-95 & 1500 & 1250 \\
\hline Punto de ablandamiento $\left({ }^{\circ} \mathrm{C}\right)$ & ASTM D 36-95 & $47^{\circ} \mathrm{C}$ & $52^{\circ} \mathrm{C}$ \\
\hline Punto de llama e ignición $\left({ }^{\circ} \mathrm{C}\right)$ & ASTM D 3143-98 & $235^{\circ} \mathrm{C}$ y $245^{\circ} \mathrm{C}$ & $220^{\circ} \mathrm{C}$ y $225^{\circ} \mathrm{C}$ \\
\hline
\end{tabular}

Fuente: elaboración propia.

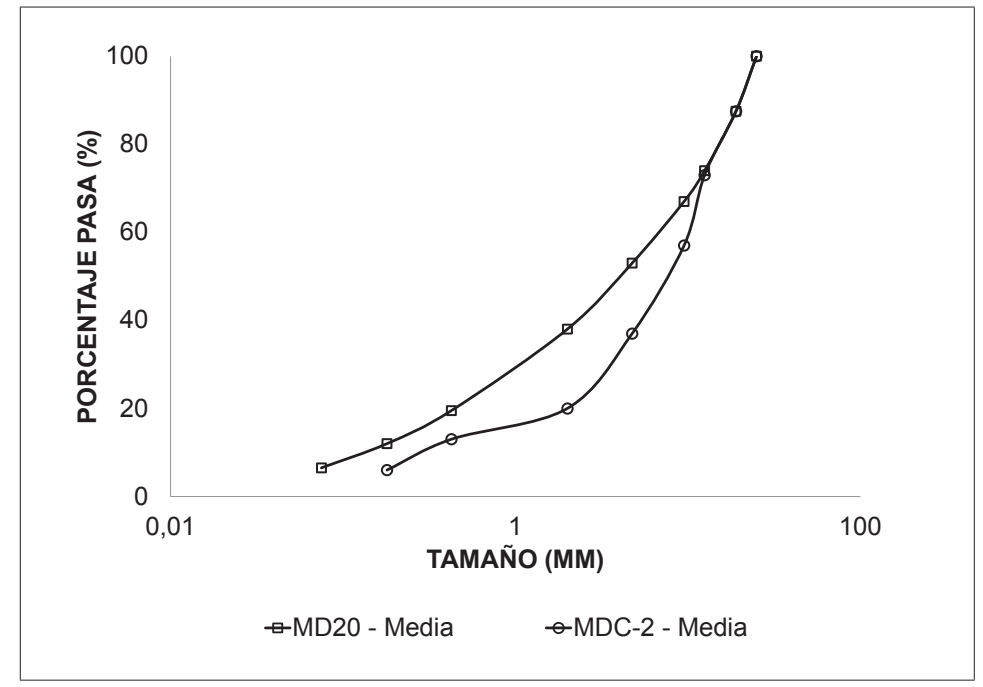

Figura 2. Curvas granulométricas MD20 de IDU y MDC- 2 del Invias

Fuente: IDU (2011) e Invias (2002). 


\section{investigación}

Tabla 2. Contenidos de asfalto en RAP

\begin{tabular}{|l|c|c|c|c|c|}
\hline \multicolumn{1}{|c|}{ Pesos de las muestras } & Muestra 1 & Muestra 2 & Muestra 3 & Muestra 4 & Muestra 5 \\
\hline Peso inicial (g) & 1200,03 & 1201,01 & 1200,13 & 1202,23 & 1200,52 \\
\hline Peso final (g) & 1126,86 & 1127,32 & 1125,98 & 1129,2 & 1123,8 \\
\hline Porcentaje asfalto (\%) & 6,10 & 6,14 & 6,18 & 6,07 & 6,39 \\
\hline
\end{tabular}

Fuente: elaboración propia.

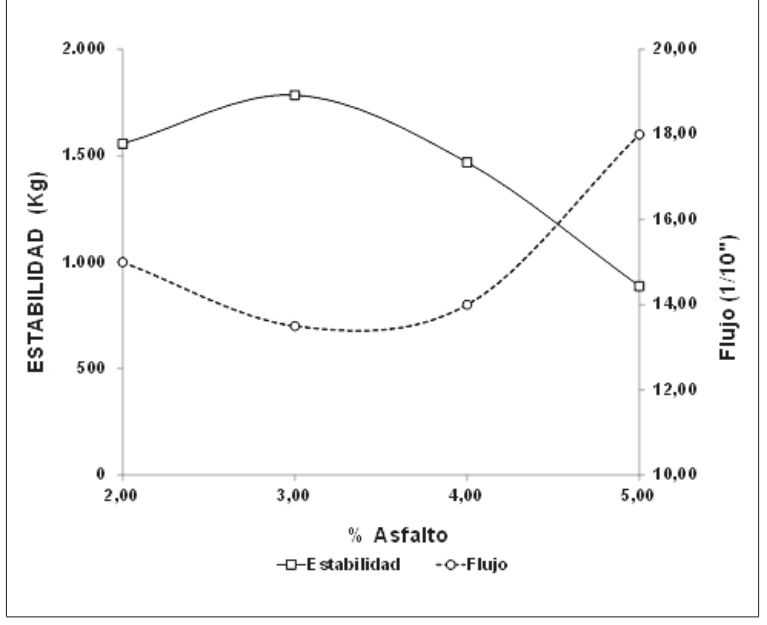

Figura 3. Estabilidad y flujo de las mezclas con $100 \%$ RAP

Fuente: elaboración propia.

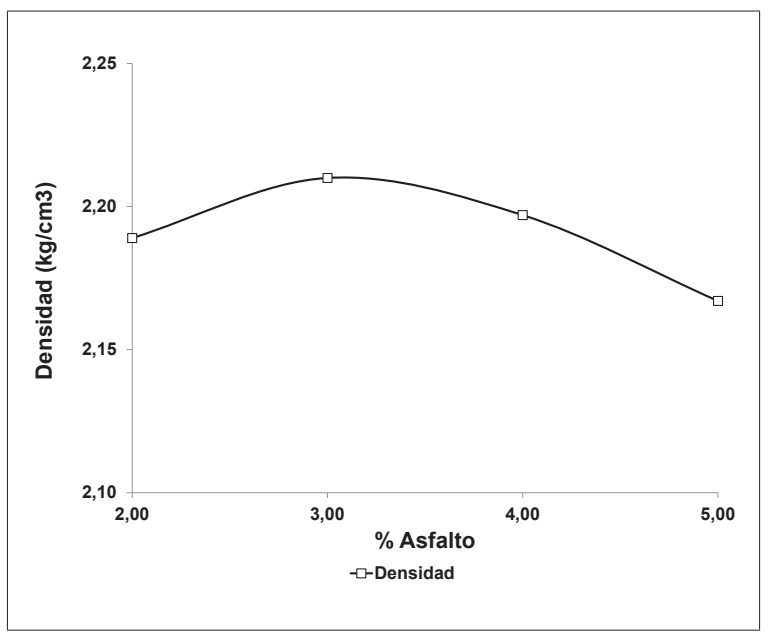

Figura 4. Densidad de las mezclas con 100\% RAP

Fuente: elaboración propia.

\section{Ensayos de fatiga}

Los ensayos a fatiga de las mezclas asfálticas fabricadas con las granulometrías MD20 y MDC-2, los asfaltos de penetración 60/70 y 80/100 y el RAP al $100 \%$ se realizaron en primera instancia con la evolución de la carga con los ciclos, estableciendo el criterio clásico de falla al $50 \%$ de la carga inicial. Además, se tuvieron en cuenta los factores de corrección por las variaciones en las dimensiones de las probetas (base mayor, base menor y altura). Se obtuvieron los siguientes resultados:

En la figura 5 se observa la evolución de la carga con los ciclos en el ensayo a fatiga, de la mezcla MD20 con asfalto 60/70, a tres diferentes niveles de deformación $(500,450$ y $300 \mu \mathrm{m})$. De las curvas de carga se evidenció que en los primeros ciclos su comportamiento es muy similar, con una pendiente constante y casi horizontal. Pasado un nivel de ciclos, la carga decrece de manera brusca y se presenta el fallo. Este comportamiento es similar para los tres niveles de deformación; solo existe la diferencia en el número de ciclos del fallo, siendo que a mayor deformación se tienen menos ciclos de vida.

En las figuras 6, 7 y 8, curvas de evolución de carga contra ciclos de las mezclas con granulometría MD 20 con asfalto 80/100, MDC-2 con asfalto $60 / 70$ y MDC-2 con asfalto $80 / 100$, respectivamente, se observó el mismo comportamiento de las curvas que con granulometría MD 20 con asfalto $60 / 70$, donde en los primeros ciclos se presenta una pendiente casi horizontal y pasado un cierto grupo de ciclos, la pendiente aumenta y se 
presenta el fallo. Cada mezcla se ensayó con diferentes niveles de deformaciones.

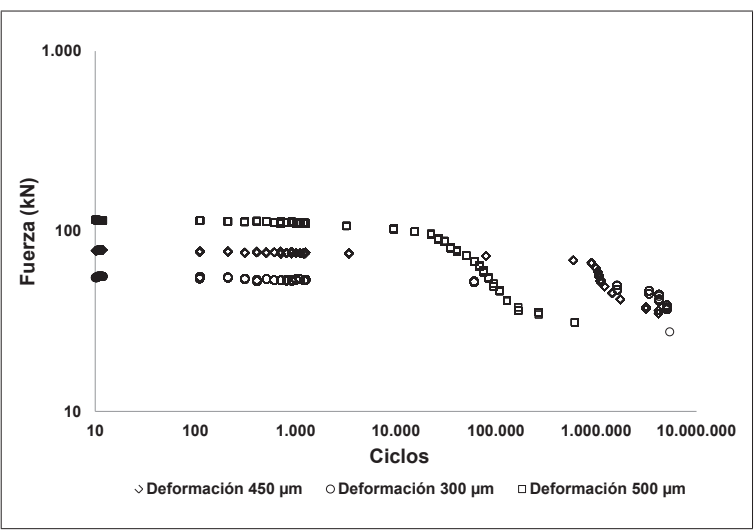

Figura 5. Ciclos versus fuerza de granulometría MD 20 con asfalto 60/70

Fuente: elaboración propia.

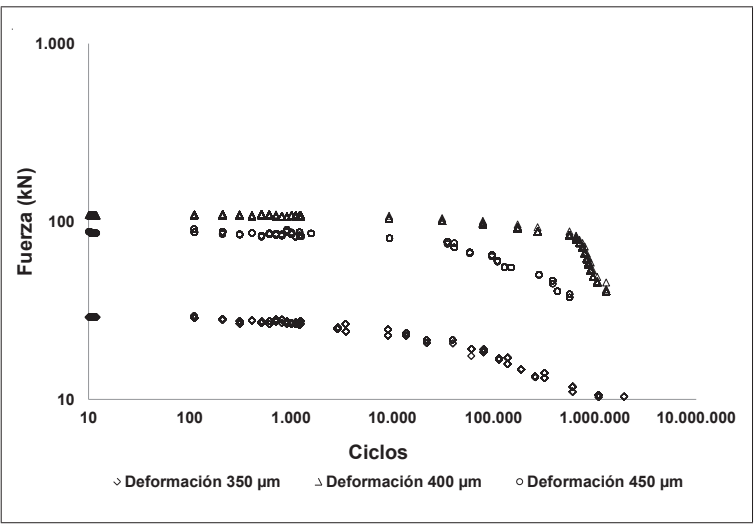

Figura 6. Ciclos versus fuerza de granulometría MD 20 con asfalto $80 / 100$

Fuente: elaboración propia.

\section{ANÁLISIS DE DATOS}

A partir de los ciclos obtenidos por el criterio clásico y la deformación correspondiente a cada ensayo, y teniendo en cuenta los factores de corrección, se obtuvieron las leyes de fatiga de cada granulometría del Invias y del IDU con sus respectivos asfaltos de penetración 60/70 y $80 / 100$.

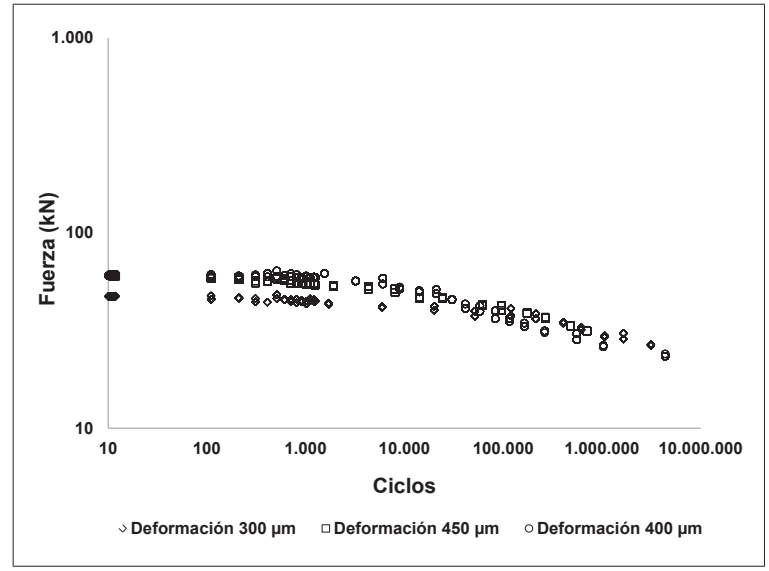

Figura 7. Ciclos versus fuerza de granulometría MDC-2 con asfalto 60/70

Fuente: elaboración propia.

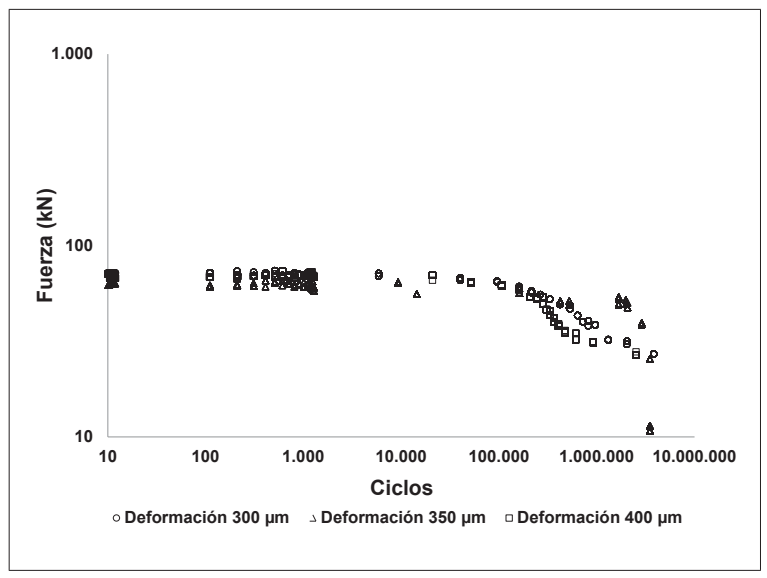

Figura 8. Ciclos versus fuerza de granulometría MDC-2 con asfalto $80 / 100$

Fuente: elaboración propia.

En la figura 9 se observan las leyes de fatiga de las mezclas con granulometría MDC-2 del Invias y asfaltos 60/70 y 80/100, donde se evidencia que los ciclos de carga son diferentes a un mismo nivel de deformación. Se observa que para una deformación dada, la mezcla con asfalto 80/100 soportó un número mayor de ciclos de carga. Por tal motivo está mezcla tiene un mejor comportamiento a fatiga. 


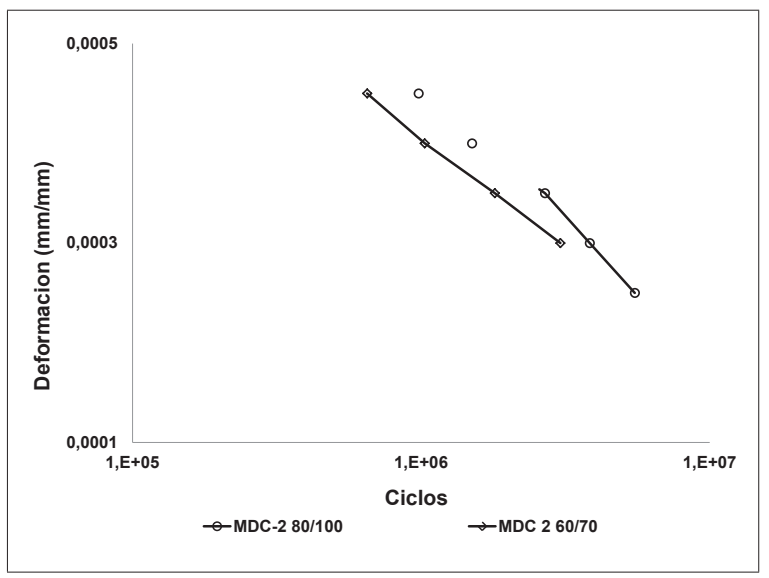

Figura 9. Leyes de fatiga de mezclas con granulometría MDC-2 del Invias y asfaltos 60/70 y $80 / 100$

Fuente: elaboración propia.

En la figura 10, leyes de fatiga de las mezclas con granulometría MD-20 del IDU y asfaltos 60/70 y $80 / 100$, se analiza que el comportamiento a fatiga de las dos mezclas asfálticas es muy similar en cuanto a su pendiente y ciclos de fallo; sin embargo, el asfalto 80/100 presentó un mejor comportamiento, al soportar un mayor número de ciclos de carga a una deformación dada.

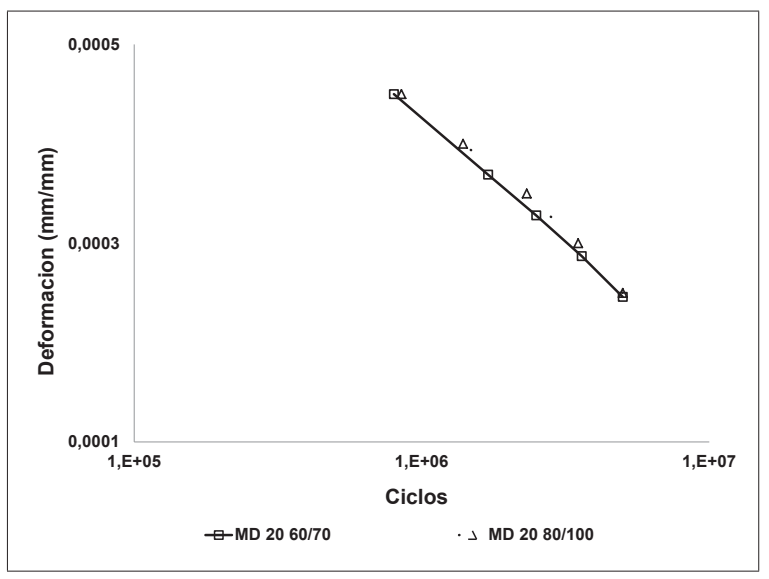

Figura 10. Leyes de fatiga de mezclas con granulometría MD20 del IDU y asfaltos 60/70 y $80 / 100$

Fuente: elaboración propia.
De la figura 11, leyes de fatiga de mezclas con granulometría MD20 del IDU y MDC-2 del Invias con asfalto de penetración 60/70, se puede observar que a menores deformaciones los ciclos tienden a ser similares, pero a valores grandes existe una diferencia significativa, lo cual muestra que la granulometría MD20 soportó mayor número de ciclos; esto evidencia, además, que la granulometría afecta el comportamiento a grandes deformaciones.

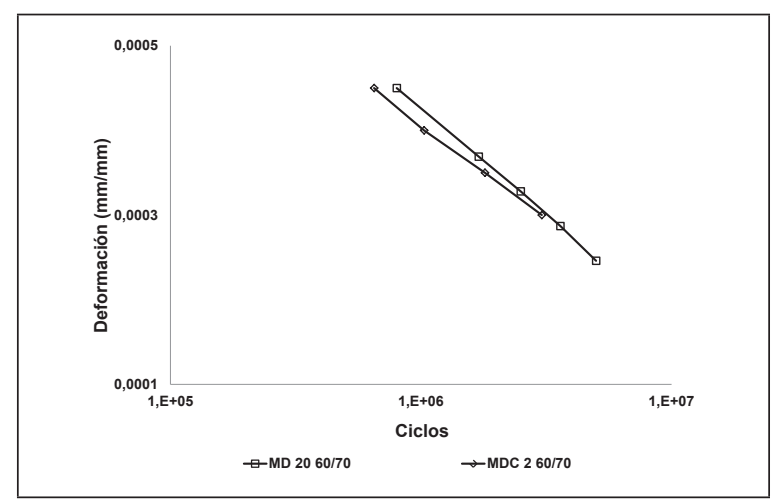

Figura 11. Leyes de fatiga de mezclas con granulometría MD 20 del IDU y MDC-2 del Invias con asfalto de penetración 60/70

Fuente: elaboración propia.

De la figura 12 se observa las leyes de fatiga de mezclas con granulometría MD 20 del IDU y MDC-2 del Invias con asfalto de penetración 80/100, donde los ciclos de carga son muy similares a una misma deformación. Se observa que con el asfalto 80/100 el comportamiento a fatiga no varía mucho para las diferentes granulometrías; sin embrago, se presentan mayores ciclos de vida en la granulometría MD 20.

En la figura 13 se observan todas leyes de fatiga estudiadas, mezclas con granulometría MD20 del IDU y MDC-2 del Invias con los asfaltos de penetración 60/70 y 80/100, donde se presenta una similitud de pendientes en las leyes; sin embargo, la granulometría MDC-2 con asfalto 80/100 tiene mayores ciclos de vida a una deformación dada. Además, la mezcla MDC-2 con asfalto 60/70 presenta la menor resistencia de ciclos de carga; por tanto, se 


\section{investigación}

evidencia que el comportamiento a fatiga no se debe solamente al tipo de granulometría, sino también al tipo de asfalto utilizado. Por otra parte, al comparar todas las leyes de fatiga con respecto a una mezcla asfáltica md20 sin RAP y asfalto 60/70, se evidencia que las mezclas con RAP, con independencia de su granulometría y tipo de asfalto son más rígidas y tienen pendientes mayores. Sin embargo, es importante resaltar que el incremento de las pendientes no es muy elevado, dadas las condiciones del uso de RAP al $100 \%$ y que la afectación es menor cuando se emplea asfalto de penetración 80/100.

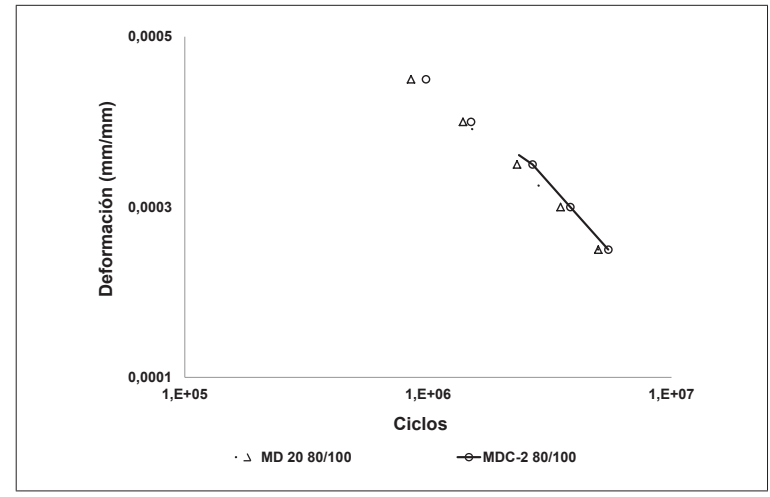

Figura 12. Leyes de fatiga de mezclas con granulometría MD 20 del IDU y MDC-2 del Invias con asfalto de penetración 80/100

Fuente: elaboración propia.

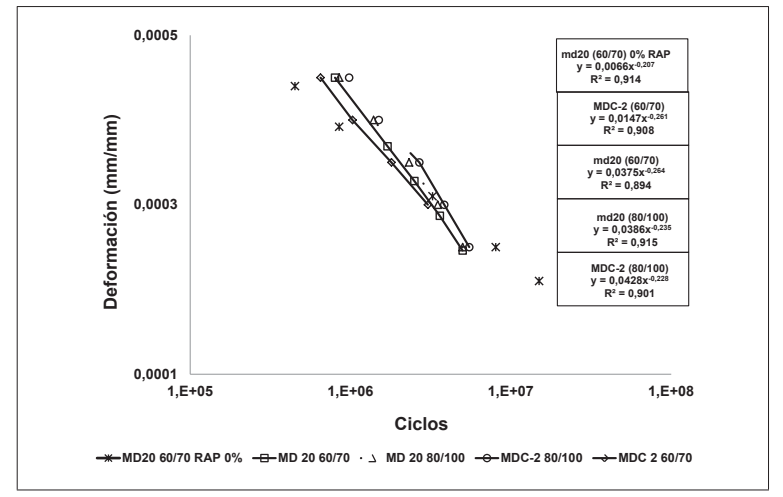

Figura 13. Leyes de fatiga de mezclas con granulometría MD 20 del IDU y MDC-2 del Invias con asfalto de penetración 60/70 y 80/100

Fuente: elaboración propia.

\section{CONCLUSIONES}

De las muestras ensayadas a fatiga con reemplazo del material granular por RAP al $100 \%$, con granulometrías MD 20 del IDU y MDC-2 del Invias y fabricadas con asfaltos de penetración $60 / 70$ y $80 / 100$, se puede concluir:

La granulometría MDC-2 con asfalto 80/100 obtuvo el mejor comportamiento a fatiga de las mezclas estudiadas, ya que al tener mayor contenido de finos en su granulometría y ser mezclado con un asfalto blando, proporcionó a la mezcla una mayor trabajabilidad; por tanto, esto generó en la mezcla mayores ciclos de vida.

Sin embargo, el mejor comportamiento a fatiga no se debe solamente al tipo de la granulometría; las mezclas son susceptibles al tipo de asfalto utilizado. Asimismo, no se puede definir cuál de las granulometrías estudiadas tiene el mejor comportamiento a fatiga, ya que en función del tipo de asfalto tienen pendientes similares.

Con el asfalto de penetración $80 / 100$ se obtuvo la mejor resistencia a fatiga para las muestras fabricadas con ambas granulometrías estudiadas, ya que al ser este un asfalto menos viscoso que el asfalto de penetración $60 / 70$, presenta menor rigidez y tiene mayor capacidad de deformación.

En el estudio comparativo de todas las leyes de fatiga con respecto a una mezcla asfáltica MD 20 sin RAP y asfalto 60/70, se evidencia que las mezclas con RAP, con independencia de su granulometría y tipo de asfalto, son más rígidas y presentan mayores pendientes. Sin embargo, es importante resaltar que el incremento de las pendientes no es muy elevado, debido a las condiciones del uso de RAP al $100 \%$ y a que la afectación es menor cuando se emplea asfalto de penetración 80/100.

Finalmente, se concluye que es posible utilizar pavimento reciclado al $100 \%$ para las mezclas 
asfálticas del estudio. Sin embargo, se deben hacer investigaciones adicionales con otras granulometrías y asfaltos modificados, con el objeto de establecer su viabilidad, no solo en bases asfálticas, sino también en carpetas de rodadura.

\section{AGRADECIMIENTOS}

Los autores agradecen a la Universidad Militar Nueva Granada, en especial al Grupo Geotecnia y Vicerrectoría de Investigaciones, por el apoyo recibido por el proyecto IMP-ING 1575.

\section{REFERENCIAS}

Abakovic, A., Gibney, A., McNally, C. y Cilchrist, M. (2010). Influence of Recycled Asphalt Pavement on Fatigue Performance of Asphalt Concrete Base Course. Journal Materials Civil Engeenering, 22 (6), 643-650.

American Society for Testing and Materials (ASTM) (2013). Penetración, ductilidad, viscosidad, punto de ablandamiento y punto de llama y ignición. Annual Book ASTM Standars, American Society for Testing and Materials.

Chen, J., Chu, P. y Lin, Y. (2007). Characterization of Binder and Mix Properties to Detect Reclaimed Asphalt Pavement Content in Bituminous Mixtures. Canadian Journal of Civil Engineering, 34 (5), 581-588.

Colbert, B. y Zhanping, Y. (2012). The Determination of Mechanical Performance of Laboratory Produced Hot Mix Asphalt Mixtures Using Controlled RAP and Virgin Aggregate Size Fractions. Construction and Building Materials, 26, 655-662.

European Asphalt Pavement Association (EAPA) (2008). Arguments to Stimulate the Government to Promote Asphalt Reuse and Recycling. EAPA, 14.

Hassan, R. (2009). Feasibility of Using High RAP Contents in Hot Mix Asphalt. Proceedings of the $13^{\text {th }}$ International Flexible $\mathrm{Pa}$ vements Conference, octubre de 2009.
Instituto de Desarrollo Urbano (IDU) (2011). Especificaciones técnicas generales de materiales y construcción para proyectos de infraestructura vial y de espacio público de Bogotá. IDU-ET-2011. Bogotá: IDU.

Instituto Nacional de Vías (Invias) (2007). Especificaciones generales de construcción para carreteras. Editorial Escuela Colombiana de Ingeniería.

Marantzidis, I. y Gidado, K. (2011). The Maximization of the Use of Reclaimed Asphalt Pavement (RAP) in the Greek Construction Industry. Proceeding 27th on the Association of Researchers in Construction, septiembre de 2011 (pp. 1125-1134).

Miró, R., Valdés, G., Martínez, A., Segura, P. y Rodríguez, M. (2011). Evaluation of High Modulus Mixture Behaviour with High Reclaimed Asphalt Pavement (RAP) Percentages for Sustainable Road Construction. Construction and Building Materials 25, 3854-3862.

Newcomb, D., Brown E. y Epps, J. (2007). Designing HMA Mixtures with High RAP Content - A Practical Guide. National Asphalt Pavement Association and US Department of Transportation FHA, Quality Improvement Series 124.

Nguyen, H. (2013). Effects of Mixing Procedures and Rap Sizes on Stiffness Distribution of Hot Recycled Asphalt Mixtures. Construction and Building Materials, 47, 728-742. 


\section{investigación}

Pérez, I., Gallego, J., Toledano, M., Taibo, J. y Garrido, F. (2007). Caracterización del comportamiento a fatiga de mezclas bituminosas en caliente fabricadas con áridos reciclados. Anales de la Mecánica de Fractura, 1, 157-162.

Reyes, O., Camacho, J. y Vargas, G. (2012). Uso de pavimento reciclado en altas tasas en mezclas asfálticas colombianas. 8 Jornadas Internacionales del asfalto y 5 semianrio latinoamericano del asfalto. Corasfaltos (pp. 1-14), octubre de 2012.

Shu, X., Huang, B. y Vukosavljevic, B. (2008). Laboratory Evaluation of Fatigue Characteristics of Recycled Asphalt Mixture. Construction and Building Materials, 22, 1323-1330.

Silva, H. (2012). Are Totally Recycled Hot Mix Asphalts a Sustainable Alternative for
Road Paving? Journal Resources, Conservation and Recycling, 60, 38-48.

Tabaković, A., Gibney, A., Gilchrist, M. y McNally, C. (2006). Fatigue Resistance of Bituminous Layers Incorporating Reclaimed Asphalt Pavement. $5^{\text {th }}$ International Conference on Research \& Practical Applications Using Wastes \& Secondary Materials in Pavement Engineering, febrero de 2006.

Valdés, G., Pérez-Jiménez, F., Miró, R., Martínez, A. y Botella, R. (2011). Experimental Study of Recycled Asphalt Mixtures with High Percentages. Construction and Building Materials, 25 (3), 1289-1297.

Widyatmoko, I. (2008). Mechanistic-Empirical Mixture Design for Hot Mix Asphalt Pavement Recycling. Construction and Building Materials, 22, 77-87. 